\title{
MULTIVARIABLE EXPANSION OF SOLUTIONS OF LINEAR EQUATIONS WITH SLOWLY VARYING COEFFICIENTS
}

BY L. E. LEVINE AND W. C. OBI

Communicated by Alberto P. Calderón, April 8, 1976

0. Introduction. This note concerns the study of the equation

$$
L_{S V}[y(t ; \epsilon)] \equiv a(\epsilon t ; \epsilon) \frac{d^{2} y}{d t^{2}}+\epsilon b(\epsilon t ; \epsilon) \frac{d y}{d t}+c(\epsilon t ; \epsilon) y=0,
$$

subject to the initial conditions

$$
y(0 ; \epsilon)=\gamma, \quad y^{\prime}(0 ; \epsilon)=\delta
$$

where $\epsilon$ is a small parameter such that $0 \leqslant \epsilon \leqslant \epsilon_{0}$ for some $\epsilon_{0}>0$, while $\gamma$ and $\delta$ are constants independent of $\epsilon$. We present a method for multivariable expansions of the solutions of this problem which is a generalization of the technique employed by E. L. Reiss [1] in the treatment of the problem

$$
\begin{gathered}
L[y(t ; \epsilon)] \equiv y^{\prime \prime}+2 \epsilon y^{\prime}+y=0, \quad t>0 \quad\left(^{\prime}=d / d t\right), \\
y(0 ; \epsilon)=0, \quad y^{\prime}(0 ; \epsilon)=1 .
\end{gathered}
$$

An important contribution of our study is the discovery of a method for systematically generating time scales from the differential equation itself.

1. Derivation of time scales. Consider an interval $D$ which includes $t=0$ and impose the following conditions:

(C.1) The functions $a, b, c$ are analytic in $D$.

(C.2) The functions $a, b, c$, do not change sign in $D$. In particular, $a$ and $c$ are never zero in $D$.

$$
t_{j}=t_{j}(\epsilon t ; \epsilon) ; \quad \frac{d t_{j+1}}{d t}=O\left(\epsilon \frac{d t_{j}}{d t}\right) \quad \text { uniformly in } D .
$$

The last condition insures that each time scale is to be "slower" than the preceding one.

It is assumed that the multivariable expansions yield "generalized uniform asymptotic expansions" in the sense defined below.

DEFinition. Let $\left\{S_{j}(t ; \epsilon)\right\}$ be a sequence of functions defined for $t \in D$ and $0 \leqslant \epsilon \leqslant \epsilon_{0}$ for some $\epsilon_{0}>0$, and let $\left\{f_{i}(\epsilon)\right\}, \epsilon \rightarrow 0$, be an asymptotic sequence. Then $\Sigma_{j=0}^{M} S_{j}(t ; \epsilon)$ is a generalized uniform asymptotic expansion of

AMS (MOS) subject classifications (1970). Primary 34E05, 34E10; Secondary 41 A60. 
$y(t ; \epsilon)$ in $D$ to $(M+1)$ terms if the remainders $R^{k}(t ; \epsilon)$ satisfy

$$
R^{k}(t ; \epsilon) \equiv y(t ; \epsilon)-\sum_{j=0}^{k} S_{j}(t ; \epsilon)=O\left(f_{k+1}\right)
$$

for each $k \in\{0,1, \ldots, M\}$.

To derive the time scales let

$$
y=y^{0}\left(\tau ; t_{0}, \ldots, t_{N}\right)+R^{0}(t ; \epsilon)
$$

where $\tau=\epsilon t$ and $R^{0}(t ; \epsilon)=O(\epsilon)$. Next, postulate the form of $y^{0}\left(\tau ; t_{0}, \ldots, t_{N}\right)$ :

I. If $a>0, c>0, b \geqslant 0$ (or $b \leqslant 0$ ), then let

$$
\begin{aligned}
y^{0}\left(\tau ; t_{0}, \ldots, t_{N}\right)= & e^{-\left(t_{1}+t_{3}+\ldots\right)} \\
& \times\left[A e^{i\left(t_{0}+t_{2}+\ldots\right)}+B e^{-i\left(t_{0}+t_{2}+\ldots\right)}\right] .
\end{aligned}
$$

II. If $a>0, c<0, b \geqslant 0$ (or $b \leqslant 0$ ), then let

$$
\begin{aligned}
y^{0}\left(\tau ; t_{0}, \ldots, t_{N}\right)= & e^{-\left(t_{1}+t_{3}+\ldots\right)} \\
& \times\left[A e^{\left(t_{0}+t_{2}+\ldots\right)}+B e^{-\left(t_{0}+t_{2}+\ldots\right)}\right] .
\end{aligned}
$$

In (6) and (7) $A$ and $B$ are constants to be determined from initial conditions. They are independent of $\epsilon$.

Substituting (5), (6) or (7) into (1) and setting the coefficients of $\epsilon^{j}$ to zero for $j=1, \ldots, N+1$, leads to equations for the time scales. All the scales are determined by initial value problems involving first order linear ordinary differential equations. In general,

$$
d t_{k} / d t=(\epsilon / 2)^{k} g_{k}(\tau ; \epsilon) ; \quad t_{k}(0)=0
$$

where the $g$-functions are determined recursively.

2. Expansions. We seek expansions of the form

$$
y=\sum_{j=0}^{M} \epsilon^{j} y^{j}\left(\tau ; t_{0}, \ldots, t_{N}\right)+R^{M}(t ; \epsilon),
$$

where $R^{M}(t ; \epsilon)=O\left(\epsilon^{M+1}\right)$ and the scales have been determined as in $\S 1$. After substituting (9) into (1), the coefficients $y^{j}$ are obtained by setting the coefficients of $\epsilon^{l}, l=0,1, \ldots, M+N$, to zero. This leads to partial differential equations for the coefficients. The solutions for these equations are generally indeterminate but they are made determinate when we substitute (9) into (2) and set the coefficients of powers of $\epsilon$ to zero as far as we can go. 
The expansions are generalized uniform asymptotic expansions in the sense of (4) and can be shown to be uniformly valid in the interval $D$. For Case I we have the following

THEOREM. If the time scales are given by (8), then for $N \geqslant 1,(9)$ is a $(M+1)$-term- $(N+1)$-time generalized uniform asymptotic expansion of the solution of (1) and (2) in the interval $D$. The error $R^{M}(t ; \epsilon)$ satisfies the inequalities

$$
\left|R^{M}(t ; \epsilon)\right| \leqslant \begin{cases}\left|Y_{\mathrm{e}}^{M}\right| \times\left\|U_{2}\right\|+B / \epsilon, & \text { if } M \text { is even } \\ \left|Y_{\mathrm{O}}^{M}\right| \times\left\|U_{2}\right\|+B / \epsilon, & \text { if } M \text { is odd } .\end{cases}
$$

The quantities in (10) are defined as follows:

$$
\begin{gathered}
Y_{\mathrm{e}}^{M}=\gamma \sum_{0 \leqslant s \leqslant[(N-1) / 2]} \prod_{2 s+1} \epsilon^{M+(2 s+1)}+\delta \sum_{1 \leqslant r \leqslant[N / 2]} \prod_{2 r} \epsilon^{M+2 r}, \\
Y_{0}^{M}=\delta \sum_{0 \leqslant s \leqslant[(N-1) / 2]} \bigwedge_{2 s+1} \epsilon^{M+(2 s+1)}+\gamma \sum_{1 \leqslant r \leqslant[N / 2]} \bigwedge_{2 r} \epsilon^{M+2 r}
\end{gathered}
$$

and $[x]$ denotes the greatest integer less than or equal to $x$. In (11) and (12) $\Pi_{i}$ and $\bigwedge_{i}$ are constants.

$U_{2}$ is the principal solution of

$$
L[y]=y^{\prime \prime}+\frac{\epsilon b(\epsilon t ; \epsilon)}{a(\epsilon t ; \epsilon)} y^{\prime}+\frac{c(\epsilon t ; \epsilon)}{a(\epsilon t ; \epsilon)} y=0
$$

satisfying $U_{2}(0)=0, U_{2}^{\prime}(0)=1$. The number $B$ is given by

$$
B=\left\|\int_{0}^{\tau=\epsilon t} G^{*}(\tau, \sigma ; \epsilon) \frac{r^{M}(\sigma ; \epsilon)}{a(\sigma ; \epsilon)} d \sigma\right\|
$$

where $G^{*}(t, s ; \epsilon)$ is the Green's function for the operator $L[y]$ and $r^{M}$ is given by

$$
L_{S V}\left[R^{M}\right] \equiv r^{M}(\tau ; \epsilon)=-\sum_{l=M+N+1}^{M+2+N} \epsilon^{l}\left(\sum_{j+k=l ; 0 \leqslant j \leqslant M} Z_{k}^{j}\right) .
$$

Finally $\|\cdot\|=\max _{t \in D}|\cdot|$.

It is to be observed that the bounds on the error in (10) are expressed explicitly in terms of the initial conditions $\gamma$ and $\delta$. Three lemmas are needed to prove the theorem; these lemmas give the detailed relationship between the coefficients, the number of time scales used, the number of terms in the expansion and the initial conditions. Applications of the results have been made in a number of specific examples including some well-known classical problems of perturbation theory [2]. 


\section{REFERENCES}

1. E. L. Reiss, On multivariable asymptotic expansions, SIAM Rev. 13 (1971), 189196. MR $44 \# 7060$.

2. W. C. Obi, A diminishing error approach to multivariable expansions of second order linear differential equations, Doctoral thesis, Stevens Inst. of Technology, Hoboken, N. J., 1975.

DEPARTMENT OF MATHEMATICS, STEVENS INSTITUTE OF TECHNOLOGY, HOBOKEN, NEW JERSEY 07030

DEPARTMENT OF MATHEMATICS AND DAVIDSON LABORATORIES, STEVENS INSTITUTE OF TECHNOLOGY, HOBOKEN, NEW JERSEY 07030 